\title{
Conocimientos sobre hidatidosis en usuarios de farmacia comunitaria
}

\author{
Francisco Guardia Sáez ${ }^{1}$, Ana Isabel Moreno Maqueda² \\ 1. Farmacéutico comunitario en Rielves (Toledo). 2. Farmacéutica comunitaria en Almendral de la Cañada (Toledo).
}

\section{PALABRAS CLAVE}

Hidatidosis, farmacia comunitaria, campaña sanitaria, educación para la salud, farmacia rural

\section{ABREVIATURAS}

DE: desviación estándar SEFAC: Sociedad Española de Farmacia Familiar y Comunitaria

SEFAR: Sociedad Española de Farmacia Rural

\section{KEYWORDS}

Hydatidosis, community pharmacy, health campaign, health education, rural pharmacy

\section{RESUMEN}

Introducción: La hidatidosis es una grave zoonosis que puede ser prevenida con métodos sencillos y con programas de educación para la salud en los que la farmacia comunitaria juega un papel clave para la realización de campañas sanitarias.

Objetivo: Saber el grado de conocimiento de las personas sobre las características de la hidatidosis y las medidas profilácticas y de control de esta zoonosis. Diseñar un proyecto educativo desde la farmacia para prevenirla, en colaboración con otros agentes sanitarios.

Material y método: Se realiza un estudio observacional con usuarios de dos farmacias. Se reclutaron personas que estaban en contacto con perros que cumplimentaron un cuestionario con objeto de registrar sus características y grado de conocimiento sobre la hidatidosis.

Resultados: Se realizaron 99 encuestas, $76(76,8 \%)$ eran propietarios de 162 perros, de los que 46 propietarios $(60,5 \%)$ los tenían como animales de compañía. $66(86,8 \%)$ los desparasitaban, la mayor parte, $32(48,5 \%)$, cada tres meses. 30 (45,5\%) desconocían el producto utilizado y $6(9,1 \%)$ utilizaban mebendazol, no aconsejado para la desparasitación.

$51(51,5 \%)$ no había oído hablar de la hidatidosis, $69(69,7 \%)$ desconocían que afecta a humanos y $88(88,9 \%)$ no sabían cómo prevenirla.

Conclusiones: Aunque el grado de desparasitación de los cánidos es aceptable, existe un elevado desconocimiento por parte de los encuestados sobre la enfermedad, métodos de transmisión y sus consecuencias, lo que nos lleva a proponer un programa de educación para la salud basado en el papel del farmacéutico comunitario como agente sanitario de primer nivel.

\section{Knowledge of hydatidosis in users of community pharmacies}

\section{ABSTRACT}

Introduction: Hydatidosis is a serious zoonosis, which can be prevented with simple preventive methods and health education programs in which the community pharmacy plays a key role in carrying out health campaigns to prevent this disease.

Objectives: To know the degree of knowledge of the population regarding the prophylactic and control measures of hydatidosis, and the capacity of the pharmacy as a sanitary agent for the prevention of this zoonosis, in collaboration with other health agents.

Materials and methods: An observational study with pharmacy users is conducted. The study enrolled people who were in contact with dogs. They were asked to complete a questionnaire on their characteristics and level of knowledge of hydatidosis.

Results: 99 questionnaires were used, 76 (76.8\%) interviewees owned 162 dogs; 46 (60.5\%) dog owners had them as pets. $66(86.8 \%)$ treated their dogs with antiparasitic drugs, most of them, 32 (48.5\%), every three months. 30 (45.5\%) did not know the product used and $6(9.1 \%)$ used mebendazol, not recommended as an antiparasitic drug.

$51(51.5 \%)$ had not heard of hydatidosis, 69 (69.7\%) did not know that it affects humans and $88(88.9 \%)$ did not know how to prevent it.

Conclusions: Although the level of antiparasitic dog treatment is acceptable, there is a high lack of knowledge of the disease, its methods of transmission and its consequences among the interviewees, which leads us to propose a health education plan based on the paper of the community pharmacist as first level health worker.
Financiación: ninguna.

Conflicto de intereses: ninguno.

Contribución a la autoría: los dos autores declaran haber participado por igual en el diseño y desarrollo del estudio, el análisis de los resultados y la redacción y revisión del manuscrito final.

Cite este artículo como: Guardia F, Moreno Al. Conocimientos sobre hidatidosis en usuarios de farmacia comunitaria. Farmacéuticos Comunitarios. 2018 Jun 30; 10(2):27-33. doi:10.5672/FC.2173-9218.(2018/Vol10).002.05 


\section{Introducción}

La hidatidosis. Agente causal.

Morfología y ciclo biológico

Los parásitos causales de la hidatidosis en humanos son tenias o gusanos planos, del grupo de los cestodos (Echinococcus). El ciclo biológico del parásito es de características indirecto con un hospedador definitivo, perro principalmente (aunque no se pueden descartar gatos y otros carnívoros salvajes, como el zorro y el lobo) y un hospedador intermediario, en el que se integran gran número de mamíferos herbívoros (especialmente la oveja, y en menor medida la cabra, jabalí y cérvidos salvajes) (figura 1) [1].

El hombre actúa en este ciclo como hospedador intermediario accidental, no tanto como parte del ciclo biológico (ya que es el fondo de saco del ciclo), sino por alimentar a sus perros con víscera crudas de ovinos con quistes hidatídicos, perpetuando la infestación.

En el perro la enfermedad no produce una sintomatología claramente evidente y relevante. Este hospedador definitivo puede albergar miles de vermes adultos y elimina los huevos embrionados en sus heces, contaminando los pastos, aguas y otros alimentos.

El hombre como hospedador accidental se infecta ingiriendo los huevos en el agua, fomites o en los alimentos contaminados o mediante ingestión directa por caricias o lametones de los perros. Debido a este último mecanismo, por su relación estrecha con los perros, los niños representan un grupo muy susceptible de infestación por el parásito [2].

Una vez producida la infección los huevos atraviesan inalterados el tracto digestivo hasta llegar al intestino delgado donde se produce la disolución de la cubierta de los huevos del parásito; liberándose los embriones que posteriormente penetran por la mucosa intestinal, introduciéndose por vía sistémica a través de la circulación portal y alojándose en diferentes órganos, principalmente el hígado, aunque pueden llegar al pulmón y con menos frecuencia a otros órganos como cerebro, riñones, bazo, hueso, etc. [3].

Los quistes hidatídicos tienen un crecimiento dispar en el organismo y este crecimiento depende del órgano afectado: pueden hacerlo rápidamente generando sintomas en un margen de tiempo corto, como ocurre en el pulmón; o crecer lentamente generando síntomas pasados años e incluso décadas como ocurre en el hígado. También pueden persistir en el hombre sin producir sintomas a lo largo de la vida [4].
Las actuaciones de prevención y control de la hidatidosis han de basarse en tres pilares fundamentales:

1. Impedir el acceso de los perros domésticos a vísceras crudas susceptibles de estar contaminadas con formas larvarias de Echinococcus $s p$. (control en mataderos, destrucción segura de las vísceras infectadas, eliminación adecuada de vísceras procedentes de cacerías o monterías, control del abandono de cadáveres, etc.) [5].

2. Control de los hospedadores definitivos:

- Control de animales vagabundos, más presentes en los entornos rurales.

- Tratamiento regular de los perros de riesgo (zonas rurales o de alta prevalencia del parásito, perros de trabajo: pastoreo, caza...,) y destrucción de las heces sospechosas de estar contaminadas.

3. Educación sanitaria.

\section{Situación epidemiológica}

La hidatidosis se incluyó en la lista de enfermedades de declaración obligatoria en 1982, año en el que se notificaron aproximadamente 2.000 casos. El RD 2.210/1995 por el que se creó la Red Nacional de Vigilancia Epidemiológica la clasificó como una enfermedad endémica regional.

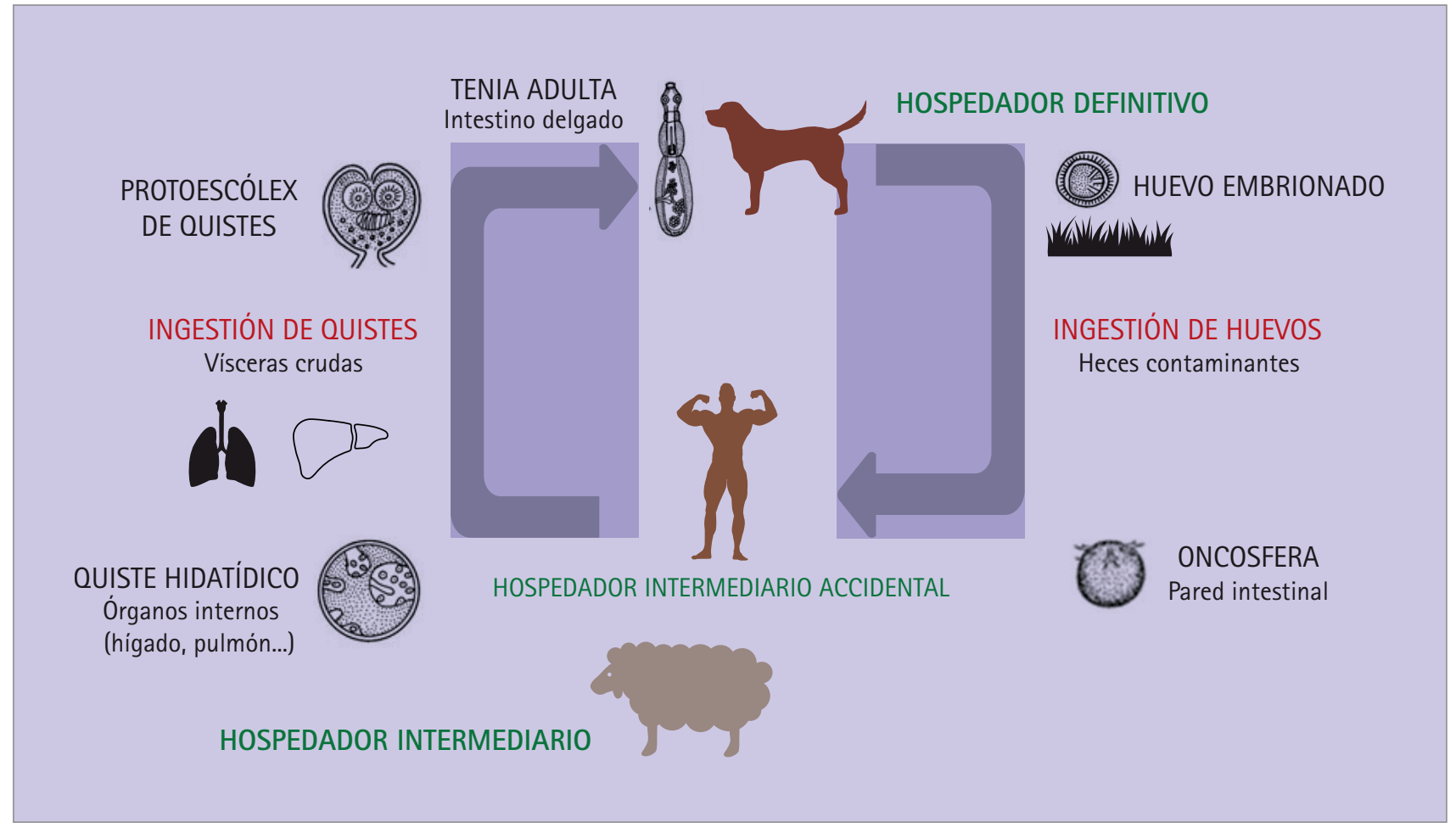

Figura 1 Ciclo biológico de la hidatidosis 
En el año 2014 se declararon 150 casos de hidatidosis en España (tasa de 0,3 casos por 100.000 habitantes), lo que supuso un ligero descenso respecto al año anterior. El número de casos fue disminuyendo hasta el año 2011 (111 casos), y aumentó en 2012 (162), manteniéndose en niveles similares desde entonces [6].

En España el principal ciclo de transmisión es un ciclo doméstico entre ganado ovino, perro y hombre. Por esta razón es más frecuente en regiones con alta producción ganadera [7].

\section{Tratamiento profiláctico}

de la hidatidosis en cánidos

El tratamiento de elección es prazicuantel. Se recomienda realizar el tratamiento periódico con prazicuantel cada 45 días en los animales de riesgo (perros pastor) y cada 120 días en los de bajo riesgo [1]. Se utiliza tanto en medicina humana como en veterinaria como antiparasitario de amplio espectro en el tratamiento de procesos originados por cestodos y trematodos. Posee una excelente actividad frente a estadíos larvarios y adultos de cestodos, afectando a la integridad y función de la membrana por alteración de la homeostasis del calcio [8]. Como marcas comerciales tenemos [9]:

- Con prazicuantel (50 mg): Zipyran ${ }^{\circledR}$, Droncid $^{\circledR}$, Canitenol-B ${ }^{\circledR}$, Overcid $^{\circledR}$, Tenecipen ${ }^{\circledR}$.

- Combinaciones con prazicuantel: normalmente se trata de combinaciones de varios principios activos:

- Combinación de prazicuantel, pirantel y febantel: Prazitel ${ }^{\circledR}$, Cestem $^{\circledR}$, Cazitel ${ }^{\circledR}$, Drontal plus ${ }^{\circledR}$, Zipyran plus ${ }^{\circledR}$, Canitenol plus ${ }^{\circledR}$, Endogard ${ }^{\circledR}$, Exitel $^{\circledR}, \mathrm{Helm} \mathrm{Ex}^{\circledR}$, Prazitel ${ }^{\circledR}$, Well-Plus ${ }^{\circledR}$, Canihelmin plus ${ }^{\circledR}$, Wormstop ${ }^{\circledR}$.

- Combinación de prazicuantel y fenbendazol: Cenfentel ${ }^{\circledR}$.

- Combinación de prazicuantel, oxantel y pirantel: Dolpac ${ }^{\circledR}$.

- Combinación de prazicuantel y milbemicina oxima: Milbemax ${ }^{\circledR}$.

- Combinación de prazicuantel y emodepside: Profender ${ }^{\circledR}$.

- Otras alternativas:

- Epsiprantel: Dosalid ${ }^{\circledR}$.

- Cestodicidas: benzimidazoles (mebendazol, fenbendazol, oxfendazol, albendazol). La actividad de los benzimidazoles sobre el género Echinococcus, actualmente es discutible por las resistencias generadas por el parásito.
Vacuna contra la hidatidosis

En algunos países (p.ej. Argentina) hay una vacuna contra la hidatidosis por Echinococcus granulosus (Providean Hidatil EG 95 ${ }^{\circledR}$ ) para bovinos, ovinos, caprinos $\mathrm{y}$ camélidos sudamericanos. Se basa en el antígeno recombinante EG95 obtenido de huevos del parásito [2], que protege frente a la infección por E. granulosus al inducir anticuerpos específicos contra la oncosfera del parásito, que es eliminado cuando ocurre la infección antes de poder establecerse en los tejidos del hospedador. Los resultados son esperanzadores. La primera dosis produce el $82 \%$ de protección, dos dosis hasta el 97\% y con tres dosis la protección es total [10]. Por el momento no existe vacuna para prevención en humanos [11].

La hidatidosis es una grave enfermedad, que puede ser prevenida con un programa de educación para la salud y unas sencillas medidas profilácticas, pero es necesario que la población sea consciente de la existencia de esta zoonosis con el objeto que adopte medidas que la eviten, entre las que destaca la desparasitación de sus perros, tanto de compañía como de ocio o de trabajo. Por ello es necesario previamente establecer el grado de conocimiento de la enfermedad por parte de la población, más si cabe la establecida en los entornos rurales.

La distribución de la red de farmacias por todo el territorio nacional, incluso en poblaciones de muy pequeño tamaño, regiones o comarcas con escasa población y muy dependientes del sector primario agrícola y ganadero [12], existiendo 932 farmacias en los 1.032 municipios españoles con poblaciones entre 500 y 1.000 habitantes; así como la preparación de los profesionales farmacéuticos, hacen de la farmacia un lugar idóneo para la realización de campañas de conocimiento, sensibilización y de educación sanitaria como es el caso de la hidatidosis, donde es básica una concienciación de la población basada previamente en el conocimiento de la existencia de la misma y las medidas profilácticas e higiénicas para evitarla.

El farmacéutico comunitario representa un papel básico, tanto en el ámbito de la educación sanitaria como instrumento preventivo de la enfermedad, como en el asesoramiento farmacológico de los propietarios de cánidos sobre las diferentes alternativas terapéuticas para el tratamiento de la infestación y prevención de la hidatidosis en los perros y su posterior contagio a humanos.

Por su proximidad y accesibilidad está plenamente capacitado para la realización de campañas de educación para la salud y de concienciación ciudadana en temas relacionados con la prevención de las enfermedades y la promoción de la salud.

Para la erradicación de la hidatidosis es preciso un conocimiento previo de esta zoonosis y de la importancia de desparasitar a los perros por los propietarios de cánidos, por los ganaderos, cazadores y la población en general para que adopten medidas que rompan el ciclo de la enfermedad y eviten su contagio.

Por ello, se ha realizado un estudio entre los usuarios de la farmacia sobre el nivel de conocimiento de esta parasitosis, previa a la posible realización de una campaña de educación para la salud en relación a la hidatidosis.

\section{Objetivo}

Evaluar el grado de conocimiento sobre la hidatidosis y sus medidas preventivas entre la población objeto de estudio.

Diseñar un proyecto educativo desde la farmacia comunitaria para prevenirla, en colaboración con otros agentes sanitarios.

\section{Material y métodos}

Estudio observacional transversal. Se realizó una encuesta durante un mes a los usuarios de dos farmacias, una situada en Rielves (Toledo) y otra en Almendral de la Cañada (Toledo) sobre su conocimiento de la hidatidosis previo a la campaña de educación para la salud.

Se entrevistó a propietarios de perros o personas en contacto con estos animales, con los siguientes criterios de inclusión:

- Ser propietario de perros o estar en contacto con ellos. Diferenciando si es mascota o es para actividades como el pastoreo o la caza.

- Todas las entrevistas son a mayores de edad de ambos sexos. 
- La actividad económica de los entrevistados se ha preguntado para determinar si están más expuestos a los factores condicionantes de la enfermedad.

Ficha de la encuesta

1. ¿Es propietario o está en contacto con cánidos? Sí/NO.

2. ¿Cuál es su actividad laboral?

3. ¿Visita habitualmente al veterinario?

4. ¿Desparasita a sus perros y si fuera positiva con que periodicidad?

5. ¿Sabe que existe una grave enfermedad que pueden contagiar los perros que puede prevenirse con un sencillo tratamiento y unas medidas higiénicas sencillas?

6. ¿Sabe que no es conveniente dar de comer a sus perros vísceras crudas de animales y que es conveniente lavar frutas y verduras antes de consumirlas, así como el lavado de manos cuando se está en contacto con cánidos?

También se valoró si sometían a sus cánidos a algún tipo de desparasitación y de ser positivo qué marca comercial utilizaban, con el objeto de valorar su idoneidad, teniendo presente la periodicidad de la desparasitación. Siendo la hidatidosis una zoonosis intimamente relacionada con la alimentación de los perros se incluyó ésta en la encuesta, así como los hábitos de lavado de frutas y verduras y el establecimiento de adquisición de las mismas.

Se utilizó una hoja de registro diseñada ad hoc en la que se recogieron también variables, edad, sexo, nivel de estudios, siempre registrando si el cánido es para uso de mascota, profesional o de ocio o ambos.

\section{Resultados}

Se realizaron 99 encuestas entre los usuarios de las dos farmacias participantes, 70 (70,7\%) hombres y $29(29,3 \%)$ mujeres, con un mayor porcentaje de jubilados con 28 $(28,3 \%)$, seguido de amas de casa con $11(11,1 \%)$. El rango de edades estuvo comprendido entre los 18-85 años con una edad media de 54,5 (DE=16,9). El tramo etario de 45-54 fue el de mayor proporción de encuestados, 46 (46,5\%). $22(22,4 \%)$ sin estudios, $48(49,0 \%)$ con estudios primarios, $14(14,3 \%)$ con estudios secundarios y $14(14,3 \%)$ con estudios superiores.
El 100\% de los entrevistados tenía contacto con cánidos, siendo dueños de perros 76 (76,8\%), con un total de 162 animales. 39 (51,3\%) solo tenían uno, teniendo el resto dos o más perros (tabla 1). La aptitud o cometido del perro se muestra en la tabla 2.

En cuanto al grado de desparasitación de los perros, 66 propietarios $(86,8 \%)$ los desparasitaban frente a 10 $(13,2 \%)$ que no lo hacían.

De los propietarios de perros desparasitados $30(45,5 \%)$ desconocían el producto utilizado. En 6 (9,1\%) casos utilizaban mebendazol antihelmíntico no aconsejado para la prevención de la hidatidosis, y el resto utilizaba un producto adecuado para su profilaxis (tabla 3).

La periodicidad de la desparasitación era cada 3 meses en 32 (48,5\%) propietarios, 13 (19,7\%) desparasitaban cada 6 meses y $12(18,2 \%)$ una vez al año (tabla 4).

En la alimentación de los cánidos, $26(34,2 \%)$ emplean piensos, $24(31,6 \%)$ piensos más alimento

Tabla 1 Número de perros por dueño

\begin{tabular}{|c|c|c|}
\hline $\begin{array}{c}\text { Perros por } \\
\text { propietario }\end{array}$ & $\begin{array}{c}\text { Número de } \\
\text { propietarios } n(\%)\end{array}$ & $\begin{array}{c}\text { Número de } \\
\text { perros } n(\%)\end{array}$ \\
\hline 1 & $39(51,3)$ & $39(24,1)$ \\
\hline 2 & $9(11,8)$ & $18(11,1)$ \\
\hline 3 & $13(17,1)$ & $39(24,1)$ \\
\hline 4 & $11(14,5)$ & $44(27,2)$ \\
\hline 5 & $2(2,6)$ & $10(6,2)$ \\
\hline 6 & $2(2,6)$ & $12(7,4)$ \\
\hline & $76(100,0)$ & $162(100,0)$ \\
\hline
\end{tabular}

Tabla 2 Aptitud o cometido del perro

\begin{tabular}{|l|c|}
\hline Aptitud & $\begin{array}{c}\text { Número de } \\
\text { propietarios } \\
n(\%)\end{array}$ \\
\hline Compañia & $46(60,5)$ \\
\hline Caza & $18(26,7)$ \\
\hline Compañia, caza & $5(6,6)$ \\
\hline Pastor otros & $2(2,6)$ \\
\hline $\begin{array}{l}\text { Pastor otros, } \\
\text { compañia }\end{array}$ & $1(1,3)$ \\
\hline Pastor ovino & $1(1,3)$ \\
\hline $\begin{array}{l}\text { Pastor ovino, } \\
\text { compañia }\end{array}$ & $1(1,3)$ \\
\hline $\begin{array}{l}\text { Pastor ovino, } \\
\text { compañia, caza }\end{array}$ & $1(1,3)$ \\
\hline $\begin{array}{l}\text { Pastor ovino, } \\
\text { pastor otros, } \\
\text { compañia }\end{array}$ & $76(100,0)$ \\
\hline & $1(1,3)$ \\
\hline
\end{tabular}

Tabla 3 Antiparasitarios usados

\begin{tabular}{|l|c|}
\hline Antiparasitario & $\begin{array}{c}\text { Número de } \\
\text { propietarios } \\
\mathbf{n}(\%)\end{array}$ \\
\hline Zipyran $^{\circledR}$ & $17(25,8)$ \\
\hline Canitenol Plus $^{\circledR}$ & $2(3,0)$ \\
\hline Dolpac $^{\circledR}$ & $2(3,0)$ \\
\hline Mebendazol $^{\circledR}$ & $6(9,1)$ \\
\hline Drontal $^{\circledR}$ & $9(13,6)$ \\
\hline No sabe & $30(45,5)$ \\
\hline & $66(100,0)$ \\
\hline
\end{tabular}

Tabla 4 Frecuencia de desparasitación

\begin{tabular}{|l|c|}
\hline Frecuencia & $\begin{array}{c}\text { Número de } \\
\text { propietarios } \\
\mathrm{n}(\%)\end{array}$ \\
\hline Cada 2 meses & $1(1,5)$ \\
\hline Cada 3 meses & $32(48,5)$ \\
\hline Cada 4 meses & $5(7,6)$ \\
\hline Cada 6 meses & $13(19,7)$ \\
\hline Cada 12 meses & $12(18,2)$ \\
\hline Cada 18 meses & $1(1,5)$ \\
\hline No sabe & $2(3,0)$ \\
\hline & $66(100,0)$ \\
\hline
\end{tabular}


Tabla 5 Tipo de alimentación de los perros

\begin{tabular}{|l|c|}
\hline Tipo de alimentación & $\begin{array}{c}\text { Número de } \\
\text { propietarios } \\
\mathrm{n}(\%)\end{array}$ \\
\hline Alimentos cocinados & $1(1,3)$ \\
\hline Pienso & $26(34,2)$ \\
\hline $\begin{array}{l}\text { Alimentos cocinados, } \\
\text { pienso }\end{array}$ & $24(31,6)$ \\
\hline Pienso, desperdicio & $23(30,3)$ \\
\hline $\begin{array}{l}\text { Alimentos cocinados, } \\
\text { pienso, desperdicio }\end{array}$ & $2(2,6)$ \\
\hline & $76(100,0)$ \\
\hline
\end{tabular}

cocinado, un 31,6\% piensos más desperdicios y $23(30,3 \%)$ pienso más desperdicios cocinados (tabla 5).

\section{Conocimiento}

de la enfermedad

$51(51,5 \%)$ de los 99 encuestados no habían oído hablar de la enfermedad, desconociendo 69 (69,7\%) que la hidatidosis afecta a los seres humanos, $86(86,9 \%)$ no conocen cómo se trasmite y $74(74,8 \%)$ cuál es el agente que la trasmite. $88(88,9 \%)$ no saben cómo prevenirla.

\section{Hábitos higiénicos}

En el lavado de frutas y verduras, $48(47,96 \%)$ limpian la fruta con agua a chorro más remojo, solo a chorro el 41,84\%, a remojo el 3,1\% y a remojo más lejía más chorro el 2,04\% (tabla 6).

En cuanto a la adquisición de las mismas el 45,45\% la adquirían en tienda más su propia cosecha, el $33 \%$ en tienda, el 13,13\% en tienda-vecino-propia y el vecino-tienda el 8,08\%.

Tabla 6 Lavado de frutas y verduras

\begin{tabular}{|l|c|}
\hline Tipo de lavado & $\begin{array}{c}\text { Número de } \\
\text { propietarios } \\
\mathrm{n}(\%)\end{array}$ \\
\hline A chorro + remojo & $42(55,3)$ \\
\hline A chorro & $26(34,2)$ \\
\hline Remojo + lejía & $4(5,3)$ \\
\hline Remojo & $3(3,9)$ \\
\hline $\begin{array}{l}\text { A chorro + remojo + } \\
\text { lejía }\end{array}$ & $1(1,3)$ \\
\hline & $76(100,0)$ \\
\hline
\end{tabular}

\section{Discusión}

El estudio que se presenta es el primero que se realizado hasta la fecha sobre el conocimiento de la hidatidosis por parte de la población usuaria de la farmacia comunitaria. En la revisión bibliográfica tampoco se han encontrado estudios similares en otros ámbitos, por lo que no podemos comparar los resultados con los obtenidos en otros estudios.

Encontramos que aunque el grado de desparasitación del los cánidos es alto llegando a cotas del 87\%, dentro de este grupo se observa que un $9 \%$ no utiliza un producto adecuado, si bien un porcentaje pequeño de usuarios que desparasita a sus perros no lo hace con la periodicidad necesaria para garantizar la correcta desparasitación. Por otra parte se ha constatado que un 63\% de los encuestados incluye en la dieta del cánido desperdicios que pueden constituir una vía de entrada para la enfermedad.

Con respecto al conocimiento de la enfermedad, vemos que el grado de desconocimiento sobre sus características es elevado, lo cual en una zoonosis de estas características es preocupante, ya que este desconocimiento puede acarrear un descuido en las medidas profilácticas encaminadas a evitar futuros contagios.

No debemos olvidar que un solo cánido infectado del parásito puede constituir por si solo una fuente muy importante de infestación de la enfermedad en las poblaciones donde se encuentre y aunque las cifras de prevalencia e incidencia de la enfermedad sean bajas, estas podrían elevarse alarmantemente en un futuro dadas las caracterizas de la evolución de la enfermedad en los individuos infectados.

Por ello es preciso, aparte de las medidas de control veterinario, establecer periódicamente campañas de conocimiento de la enfermedad y de las medidas profilácticas para posibles futuros contagios y para alcanzar este objetivo se propone como conclusión de este estudio un proyecto de educación para la salud, en el que se pretende involucrar a las instituciones sanitarias y a diferentes agentes sanitarios, entre ellos los farmacéuticos comunitarios, cuyas características y objetivos se detallan en el anexo y que podría ser liderado por alguna institución o sociedad profesional enmarcadas en el ámbito de la farmacia comunitaria.
Referencias bibliográficas

1. Zorita Moreno M. Programa control de la hidatidosis en España [Internet]. Madrid: Facultad de farmacia de la universidad de Madrid; 2015. [Consultado 12/9/17]. Disponible en: http://147.96.70.122/Web/TFG/TFG/ Memoria/MARINA\%20ZORITA\%20 MORENO.pdf

2. Ministerio de Salud. Presidencia de la Nación Argentina. Enfermedades infecciosas: hidatidosis. Guía para el equipo de Salud [Internet]. Buenos Aires: Dirección de Epidemiología Ministerio de Salud; 2012. [Consultado 19/9/17]. Disponible en: http://www. msal.gob.ar/images/stories/epidemiologia/pdf/guia-medica-hidatidosis.pdf

3. Del Carpio M, Mercapide CH. Normas de diagnostico y tratamiento de la hidatidosis humana Provincia de Rió Negro Argentina. Salud ambiental-Uresa Zona Andina [Internet]. Buenos Aires: Ministerio de Salud Argentina; 2010. [Consultado 13/9/17]. Disponible en: http://www. uresaandina.com.ar/wp-content/ uploads/2016/08/Normas_Diagnostico_Tratamiento_Hidatidosis_2010.pdf

4. Uribarren Berruela T. Hidatidosis, equinococosis o quiste hidatídico [Internet]. Departamento de microbiología y parasitología. Facultad de Medicina. México: Universidad Nacional Autónoma de México UNAM; 2015. [Consultado 12/9/17]. Disponible en: http://www.facmed.unam.mx/deptos/ microbiologia/parasitologia/hidatidosis.html

5. Laura EA. Prevención de la hidatidosis Cartillas de educación sanitaria [Internet]. Consejo Nacional de la Educación Técnica Argentina: Ministerio de Cultura y Educación; 1976. [Consultado 23/9/17]. Disponible en: http://www.bnm.me.gov.ar/giga1/documentos/EL003969.pdf

6. Amillategui Dos Santos R, Cano Portero R. Resultados de la vigilancia epidemiológica de las enfermedades trasmisibles informe anual [Internet]. Red Nacional de Vigilancia Epidemiológica Instituto de Salud Carlos III. Madrid: Ministerio de Economía y Competitividad, Ministerio de Sanidad, Servicios Sociales e Igualdad; 2014. [Consultado 15/11/17]. Disponible en: http://www.isciii.es/ISCIII/ es/contenidos/fd-servicios-cientifico-tecnicos/fd-vigilancias-alertas/ fd-enfermedades/pdf_2016/RENAVE_ INFORME_ANUAL_2014.pdf

7. Amillategui Dos Santos R, Cano Portero R. Resultados de la vigilancia epidemiológica de las enfermedades trasmisibles informe anual [Internet]. Red Nacional de Vigilancia Epidemiológica Instituto de Salud Carlos III. Madrid: Ministerio de Economía 
y Competitividad, Ministerio de Sanidad, Servicios Sociales e Igualdad; 2013. [Consultado 18/10/17]. Disponible en http://www.isciii.es/ISCIII/ es/contenidos/fd-servicios-cientifico-tecnicos/fd-vigilancias-alertas/ fd-enfermedades/Renave_informe_ anual_2013.pdf

8. Consejo General de Colegios Oficiales de Farmacéuticos [Internet]. Praziquantel en Medicina Veterinaria. Bot Plus. [Acceso 14/3/2018]. Disponible en: https://botplusweb.portalfarma.com/Documentos/2004/2/23/18640.pdf

9. Centro de Información Online de Medicamentos Veterinarios de la AEMPS - CIMA Vet. Medicamentos con princi- pio activo Prazicuantel para tratamiento de Cánidos [Internet]. Agencia Española de Medicamentos y Productos Sanitarios; 2017. [Consultado 26/9/17]. Disponible en: https://cimavet.aemps. es/cimavet/medicamentos.do

10. Red Nacional de Vigilancia Epidemiológica. Instituto de Salud Carlos III [Internet]. Protocolos de la Red Nacional de Vigilancia Epidemiológica. Protocolo de Vigilancia de Hidatidosis. 2013. [Acceso 20/2/2018]. Disponible en: http://www.isciii. es/ISCIII/es/contenidos/fd-servicios-cientifico-tecnicos/fd-vigilancias-alertas/fd-procedimientos/PROTOCOLOS_RENAVE-ciber.pdf
11. Lightowlers $M$, Jensen 0 , Fernández E, Heath D, Woollard DJ, Gauci CG, et al. Vaccination trials in Australia and Argentina confirm the effectiveness of the EG95 hydatid vaccine in sheep [Internet]. Int J Parasitol 1999; 29(4): 531-534. [Consultado 13/9/17]. Disponible en https://www.ncbi.nlm. nih.gov/pubmed/10428628

12. Guerrero FJ. La farmacia rural la esencia del modelo español de farmacia [Internet]. Sevilla: SEFAR; 2012. [Consultado 22/10/17]. Disponible en: http://cofzaragoza.org/quelinka/ privada/wp-content/uploads/2013/02/ Resumen-del-Estudio-Farmacia-Rural.pdf 


\section{Anexo \\ PROYECTO DE EDUCACIÓN SANITARIA A TRAVÉS DE LAS FARMACIAS COMUNITARIAS CON EL OBJETO DE CONCIENCIAR A LA POBLACIÓN SOBRE LOS PELIGROS DE LA HIDATIDOSIS}

\section{Nombre del proyecto \\ PREVENCIÓN Y PROMOCIÓN DE LA SALUD EN HIDATIDOSIS EN LOS ENTORNOS RURALES ESPAÑOLES A TRAVÉS DE LAS FARMACIAS COMUNITARIAS Y EN COLABORACIÓN CON LOS AYUNTAMIENTOS, VETERINARIOS DE ZONAS BÁSICAS DE SALUD Y CONSULTORIOS MÉDICOS LOCALES.}

\section{Síntesis del proyecto}

La presente propuesta pretende fortalecer los ejes salud y cuidado del medioambiente de las poblaciones españolas través de acciones divulgativas, acciones de educación para la salud y promoción de la salud, dirigidas a:

- La prevención de la hidatidosis.

- La promoción de la salud en hidatidosis.

- Evitar la contaminación del medioambiente.

\section{Fundamentación del proyecto}

La hidatidosis es una enfermedad prevenible basándose en su conocimiento y hábitos de vida adecuados. Consideramos que, a partir de la amplia distribución de la farmacia y considerando que esta enfermedad tiene más prevalencia en entornos rurales dadas sus características económicas y sociales, la farmacia comunitaria se presta, dada su amplia distribución, el conocimiento de los pacientes y la preparación del titular que ejerce en dichas farmacias, como un elemento de primer orden para asegurar la salud integral que pretendemos para la población española en general y, en concreto, para evitar esta zoonosis. Al tiempo también brindaría conocimientos para evitar la contaminación del ambiente que actúa como factor facilitador de esta zoonosis.

A partir de la confección e implementación de proyectos sanitarios y de educación para la salud se busca generar un diálogo entre la farmacia como centro sanitario y la comunidad, necesario para la planificación y logro de objetivos comunes, que no pueden ser otros que atender a las demandas de la mayoría y gestar en las farmacias un espíritu solidario que beneficie tanto a la comunidad como a los propios farmacéuticos. El presente proyecto pretende cumplir con estos objetivos.

\section{Objetivo general}

Concienciar a los beneficiarios directos e indirectos sobre hábitos de vida para la prevención de la hidatidosis a través de estrategias de intervención en el área de educación para la salud.

\section{Objetivos específicos}

- Generar espacios interactivos junto a los equipos médicos locales para el tratamiento y abordaje de la hidatidosis para que la población objeto alcance un nivel de salud y educación integral.

- Distribución de folletos y carteles con información referida a hidatidosis, formas de contagio y medidas preventivas.

- Informar a la población sobre los diferentes tratamientos antiparásitos existentes en el mercado para la desparasitación de sus perros y concienciarlos para que mantenga sus cánidos bajo un control veterinario.

- Organización de charlas educativas en escuelas rurales, centros sociales y de mayores para concienciar a la población.

- Informar a las sociedades de cazadores locales de la necesidad de desparasitar a todos sus perros y camadas y evitar alimentarles con visceras animales.

- Podría valorarse una primera distribución gratuita de tratamientos antiparásitos a los propietarios de cánidos a través de las farmacias, con el objeto de concienciarlos en la desparasitación de sus perros.

- Promover en los establecimientos ganaderos rurales y mataderos, junto con los equipos veterinarios, la eliminación de las achuras de las ovejas a través de las cuales se produce el contagio al hombre siendo el reservorio el perro y asegurar el saneamiento del ambiente.

\section{Tiempo estimado del proyecto}

1 año.

\section{Actores e instituciones que participan en este proyecto}

- Farmacéuticos, Sociedad Española de Farmacia Familiar y Comunitaria (SEFAC), Sociedad Española de Farmacia Rural (SEFAR), Colegios Oficiales de Farmacéuticos.

- Médicos, enfermeros, veterinarios y agentes sanitarios de las zonas rurales.

- Técnicos de las Consejerías de Sanidad.

- Maestros rurales.

- Organismos públicos, Ayuntamientos, Diputaciones, Gobiernos regionales.

\section{Memoria económica}

- Dípticos y folletos. Precio 0,30 € por ejemplar. 100 por localidad. $30 €$ por localidad donde se desarrollara el proyecto.

- Cartelería, póster. Precio $1 €$ por ejemplar. 6 pósteres por localidad. Total 6 euros.

- Charlas informativas, en colegios, asociaciones...., coste 0, a cargo del farmacéutico comunitario de la localidad, técnicos de la consejerías y veterinarios por determinar.

Total coste proyecto por localidad: $36 €+$ Coste charlas informativas.

También habría que incluir el coste de muestras gratuitas de antiparasitarios, si se incluyera en el programa esta acción profiláctica. 